$16^{\text {th }}$ International Conference on

AEROSPACE SCIENCES \& AVIATION TECHNOLOGY,

ASAT - 16 - May 26 - 28, 2015, E-Mail: asat@ mtc.edu.eg

Military Technical College, Kobry Elkobbah, Cairo, Egypt

Tel : +(202) 24025292 - 24036138, Fax: +(202) 22621908

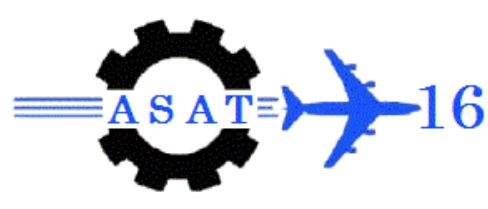

\title{
AUTOMATED INSPECTION OF ASSEMBLY PARTS USING MACHINE VISION
}

\begin{abstract}
M. El-Agamy ${ }^{*}$, M. Awad ${ }^{\dagger}$, H. Sonbol ${ }^{\ddagger}$
Abstract: Automated inspection has become an essential requirement in automated manufacturing system. The advances in computer vision and image processing contributed in enhancing developing better vision-based inspection systems that enhanced the efficiency of automated manufacturing systems. In this paper, a new vision-based inspection model for automatic inspection of assembly parts is presented. The developed model can perform different inspection functions including: measurement, counting, checking the presence of part/ features, assembly direction and proper surface coating. The model receives the part images as an input and automatically generates the inspection results and the acceptance or removal of the part either for rework or rejection.
\end{abstract}

Keywords: Visual-Based Inspection, Computer Vision, Digital Image Processing and Machine vision

\section{Introduction}

Computer vision is a modern technology that emerged and contributed in development of automated vision-based inspection systems (VBIs) [1,2]. Computer vision use image processing techniques to executes specific function or outcome based on the image analysis of part under inspection and the inspection rules set by the designer embedded into the vision model [3].

Computer vision is based on Digital image processing (DIP) techniques. Selection of proper DIP can positively contribute to the accuracy of the obtained results. DIP involves image acquisition, image pre-processing, image segmentation and edge detection, morphological operations viz. erosion, dilation, closing, opening. VBIs applications increased in manufacturing industry because they support automation, standardization, integration and higher productivity while decreasing the inspection time, cost and required inspection skills [4]. VBIs provide also more consistent and reliable inspection results as they decrease the effect of human bias or fatigue [5]

\footnotetext{
*Col. Eng., Master Degree in Mechanical Engineering /elagamy_2000@yahoo.com

${ }^{\dagger}$ Professor, Dpt. of Mechanical Engineering, Ain Shams University, Cairo, Egypt

* Professor, Dpt. of Mechanical Engineering, Ain Shams University, Cairo, Egypt
} 


\section{Literature Review}

VBIs are used in in-process inspection to control production and to achieve the desired product quality rather than a means of acceptance or rejection at the end, where faulty parts can be identified and removed earlier before further machining or assembly process. They meet the requirements of modern automated manufacturing systems in terms of the accuracy, speed, flexibility and cost efficient inspection processes. Appling VBI doesn't require high inspector expertise or fabricating special jigs or fixtures for holding the part under inspection as in traditional systems. So, they are more efficient inspection methods in terms of cost, time and automation in production process as well [6].

The typical functions in VBIs that use computer vision techniques include image acquisition and pre-processing; feature extraction, image segmentation, High-level processing and decision making. Image acquisition step produces digital image of part under inspection; image pre-processing is used for enhancing the image including noise removal, scaling and enhancing contrast; feature extraction including lines, edges and Regions of Interests (ROI); Image segmentation is used to identify the points or regions in the image relevant for further processing like selection of a specific set of interest points or segmentation of one or image regions which contain a specific object of interest; High-level processing include detection of an object size, direction, Image recognition (classifying a detected object into different categories) or Image registration (comparing and combining two different views of the same object). Finally, decision making step involves the final decision required for the application, for example Pass/fail on automatic inspection applications [7].

VBIs require proper selection and implementation of edge detection and image segmentation techniques [8]. Edge detection is used to detect significant edges of the part in the image for using as a reference in inspection tasks like measurement, counting of parts or features and verification of proper part direction in production lines. Image segmentation is used in checking the presence/ absence of parts /features, identification of proper surface coating [9] $\&[10]$.

In conventional inspection systems, a workpiece machined on a machining center requires being moved to a Coordinate Measuring Machine (CMM) to check its dimensional accuracy. The manual job set-up and inspection of machined parts are usually time consuming, subject to human errors, and often lead to longer lead times. The bottleneck problem is further compounded with the difficulty of capital investment and time delay of material flow between CMMs and machine tools in the factory. Implementing computer vision in VBI has contributed in solving this problem by in-line automated inspection systems [4].

VBIs have been proposed for inspection of parts in automotive industry. Angrisania et al. (1999), described an automatic inspection of geometrical and physical characteristics of automotive gaskets. The system measures the height, width, thickness and sponginess parameters. The system reduced inspection time, measurement uncertainty and cost. They argued that this method is more suitable for inspection flexible parts more traditional techniques because traditional methods are based on contact measurement that may either damage the product or change its size. However, the developed system accuracy needs to be valid using more test samples with different sizes [11].

Ayub et al (2014) developed a VBI system for measurement the roundness of automotive camshafts based computer vision and image processing techniques where images of the parts were collected and processed. The results showed high effectives and reliable in roundness measurement for the camshafts application [12]. However, as this research was conducted using one nominal diameter of $46 \mathrm{~mm}$ where the average roundness that could be measured 
was $0.15 \mathrm{~mm}$, so the measurement accuracy of the system need to verified using different shaft sizes. A recent application for VBI approach in manufacturing of automobile parts was presented by $\mathrm{Wu}$ et al (2015), where a non-contact method based on monocular images is used for measuring the position and attitude parameters of weld stud used in automobile industry. The method meets the requirement of online flexible and high-precision measurement of weld studs [13]. However, the accuracy of the proposed method decreases with the increase of the attitude deviation angle, improper lighting or orientations of the stud due to the decrease in acquired image quality.

VBIs were also used in general inspection application where the size or the number of the parts under inspection challenges the traditional measurement methods. For example, Lu et al (2001), presented an image based technique for measuring the straightness of large steel pipes using laser sensors. The system accuracy and stability were high [14]. Although the developed system had high accuracy, still it suffers from complexity in hardware setup, calibration, the large number of parameters that need to be considered in addition to its cost. Another example is the inspection of machining tools inserts where the size is small and quantity is high.

Schmitt et la. (2010) proposed an inline machine vision inspection system for carbide tool inserts. The system inspects insert coating colour, edge radius, plate shape and chip-former geometry. The main steps are image acquisition, segmentation, inspection of coating colour, measurement of the edge radius and classification of plate shape and chip-former geometry. The classification accuracy was $92 \%$ and the minimum edge radius was $0.18 \mathrm{~mm}$. Although this method results showed a good accuracy, However, using more samples with different types are recommended to ensure its generalization capabilities [6].

Another application of VBIs was introduced by Ali et al. (2014), for measurement of gear teeth profile. They argued that employing VBI in gear measurement eliminates limitations / disadvantages of traditional measurement systems including the risks of human injury and damaging of expensive measuring tool stylus if collided with the gear or other parts, long inspection time, high cost and low productivity and low reliability of the measurement results [15]. However, the developed system performance needs to be enhanced via using better image processing techniques by using Sobel edge detection, threshold and blob counter.

To sum up, the above literature review findings show that presented VBI methods either perform few inspection functions, mainly measurement or defect detection as in $[6,16,17]$; while not addressing other important inspection functions like verification of parts or feature presence, proper assembly guidance, counting and verification of existing features. Developing more integrated inspection systems that include many inspection functions would help in increasing the effectiveness and integration required in automated manufacturing systems.

In this research, new inline automatic inspection method (CAI-1) for assemblies parts based on computer vision techniques is proposed. CAI-1 performs the following functions: measurements, checking the presence of parts/features, counting existing parts or features and verification of proper part direction and surface coating; and generates the results with the required inspection decisions automatically.

\section{Methodology}

This section provides the main steps used to develop and implement the CAI- inspection model shown in Fig. (1). 

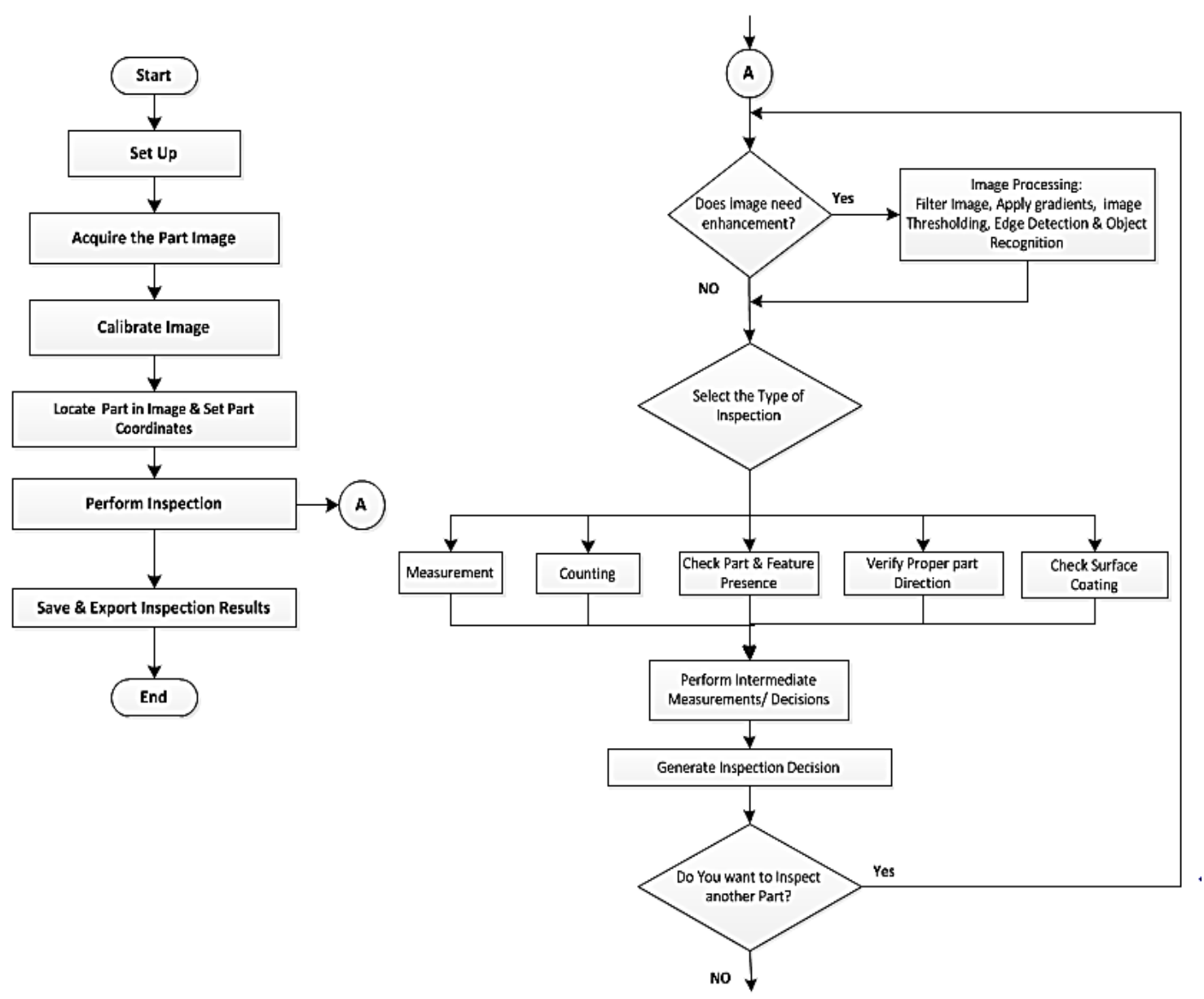

Fig.1 CAI-1 Vision Model

\subsection{Setup and Image Acquisition}

CAI-1 is started by acquisition of parts images and proceeds automatically to other image processing and inspection steps. The set up used in this work consists of the following: (1) suitable CCD positioned perpendicular to the part under inspection; (2) uniform light source that provides enough contrast between the part and background; (3) PC computer; and (4) motor-driven conveyor. Parts under the inspection are placed on the conveyor that moves while the camera is supported in parallel direction to in front of the part.

\subsection{Image Calibration}

The image is calibrated to transform the measurement units from pixel value to the real world measurements in order to be more practical and convenient to the user taking into consideration: (1) The inspection image contains little or no lens distortion; (2) The real-world distance between two distinct points in the image is known; and (3) The camera is perpendicular to the inspection surface. Calibration is made based on the selected pixel points within a specified ROI. CAI-1 uses a calibration algorithm and the selected pixel for realworld mappings to compute calibration information for the entire image to convert the pixel mapping to real-world mapping. After calibration the image, calibration axes are defined in order to express pixel measurements in real-world units.

\subsection{Locating the Part in image}

The next step is to specify the region of interest (ROI), locate the part coordinates and its origin using edge detection technique explained in the previous sections. The ROIs will be shifted and rotated to match with possible part shifts relative to the reference image. For 
moving the ROIs with the part, a reference coordinate system will be defined with relative to the object in the reference image. The coordinate system moves with the part when it is shifted and rotated in the image to cover the problem of dislocation of the parts due to their set on a moving conveyor. The part coordinates are used as the reference for further inspection and measurement steps. Fig.2 illustrates image calibration and setting part coordinates.
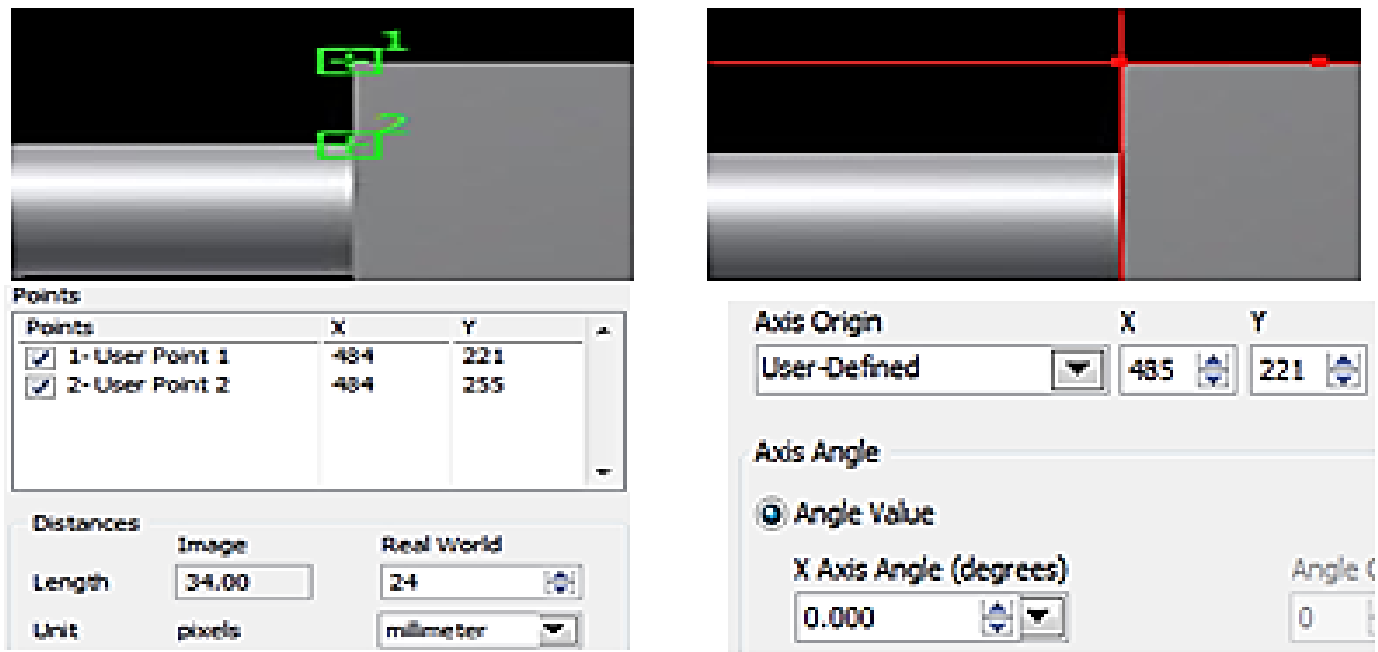

Fig.2 Locating the Part Coordinates in Image

\subsection{Image Processing for Edge Detection and Object Recognition}

This section introduce the implemented image processing for enhancing image quality, edge detection and object recognition; and essential before proceeding and executing inspection tasks.

\subsubsection{Smoothing Image}

Images taken from a camera normally contain some amount of noise that should be reduced to avoid false detected edges that can reduce the accuracy VBI system. Image intensities are smoothed by applying a Gaussian convolution linear filter to reduce the existing noise. A convolution is an algorithm that consists of recalculating the value of a pixel based on its own pixel value and the pixel values of its neighbors weighted by the coefficients of a convolution kernel. The sum of this calculation is divided by the sum of the elements in the kernel to obtain a new pixel value. The size of the convolution kernel is squared matrix (5x5) with a standard deviation of $\sigma=1.4$ as shown in Equation (1). The effect of using smoothing with Gaussian filter is illustrated in the example shown in Fig (4.b).

$$
B=\frac{1}{159}\left[\begin{array}{ccccc}
2 & 4 & 5 & 4 & 2 \\
4 & 9 & 12 & 9 & 4 \\
5 & 12 & 15 & 12 & 5 \\
4 & 9 & 12 & 9 & 4 \\
2 & 4 & 5 & 4 & 2
\end{array}\right]
$$

\subsubsection{Finding Gradients}

An edge in an image may point in a variety of directions that need to be detected. CAI-1 uses Sobel operator to detect edge strength, and directions in horizontal, vertical and diagonal directions from the blurred images. The edge strength $(G)$ and direction $(\theta)$ are calculated by equations (2\&3). 


$$
\begin{aligned}
& |G|=\left|G_{x}\right|+\left|G_{y}\right| \\
& \theta=\arctan \left(\frac{\left|G_{x}\right|}{\left|G_{y}\right|}\right)
\end{aligned}
$$

Where: Gx and Gy are the first derivatives in the horizontal and vertical directions; and $(\theta)$ is the edge angle. Fig.(4.c) show that detected edges are clear but they are relatively thick and blurred, So, further processing is made for more accurate edge detection as mentioned below.

\subsubsection{Non-maximum Suppression}

Non-maximum suppression algorithm is used to convert blurred edges to sharp (thin) edges by preserving all local maxima in the gradient image as shown in Fig. (4.d) The model scans all pixels in the gradient image and performs the following steps:

1. Round the gradient direction $\theta$ to nearest $45^{\circ}$, corresponding to the use of an 8 -connected neighborhood.

2. Compare the edge strength of the current pixel with the edge strength of the pixel in the positive and negative gradient direction. i.e., if the gradient direction is north $\left(\theta=90^{\circ}\right)$, compare with the pixels to the north and south.

3. If the edge strength of the current pixel is largest; preserve the value of the edge strength.If not, suppress (i.e. remove) the value.

Fig.3 illustrates a simple example of non-maximum suppression. The edge strengths are indicated both as colors and numbers, while the gradient directions are shown as arrows. The resulting edge pixels are marked with white borders. Pixels that have gradient directions pointing north are compared with the pixels above and below where maximal pixels are marked with white -borders while others are suppressed.

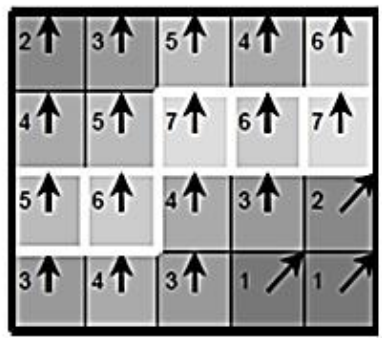

Fig.3 Non-maximum Suppression Example

\subsubsection{Double Thresholding}

Potential edges are determined by double thresholding process. The remaining edge-pixels after the non-maximum suppression will probably be true edges in the image, but some may be caused by noise or color variations for instance due to rough surfaces. To identify the true edges, thresholding is used to preserve only stronger edges with certain value. The edge detection algorithm uses double thresholding. Edge pixels stronger than the high threshold are marked as strong; edge pixels weaker than the low threshold are suppressed and edge pixels between the two thresholds are marked as weak. The effect on the test image with thresholds of 20 and 80 is shown in Fig. 4.e.

\subsubsection{Final Edge Detection}

Final edges are determined by suppressing all edges that are not connected to strong edges. Strong edges are interpreted as "certain edges", and can immediately be included in the final 
edge image. Weak edges are included only if they are connected to strong edges. The logic is that noise and other small variations are unlikely to result in a strong edge when using proper threshold levels. Thus strong edges result from true edges in the original image. The weak edges can either be due to true edges or noise/color variations. The latter type will probably be distributed independently of edges on the entire image, and thus only a small amount will be located adjacent to strong edges. Weak edges due to true edges are much more likely to be connected directly to strong edges. Edge tracking can be implemented by BLOB-analysis (Binary Large Object). The edge pixels are divided into connected BLOB's using 8-connected neighborhoods. BLOB's containing at least one strong edge pixel are then preserved, while other BLOB's are suppressed. The effect of edge tracking on the test image is shown in Fig.4.

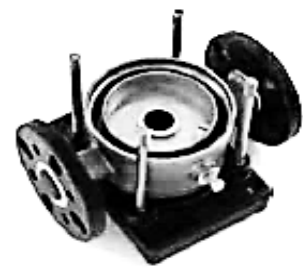

(a) Original

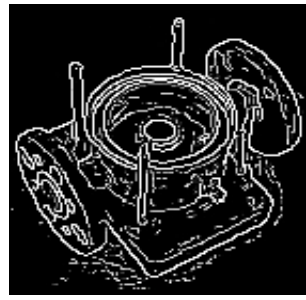

(e) Double thresholding

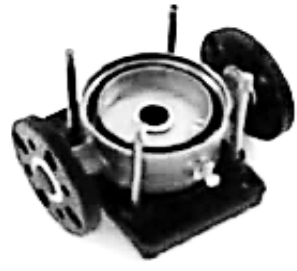

(b) Smoothed

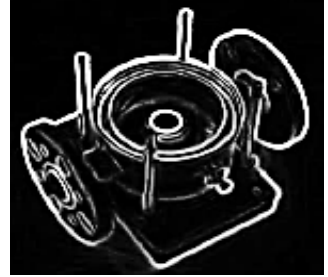

(c) Gradient Magnitudes

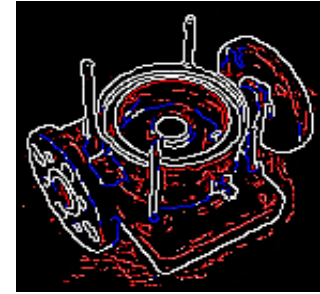

(f) Edge tracking by hysteresis

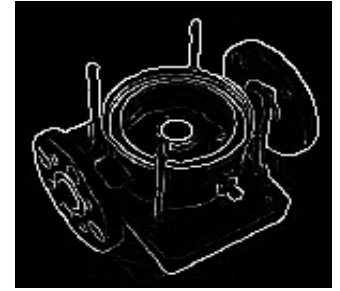

(d) Edge after non-maximum suppression

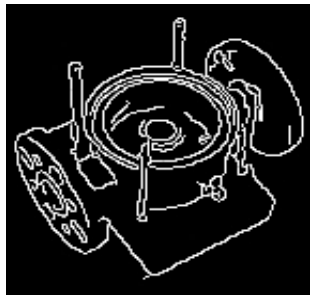

(g) Final Output

Fig.4 Edge Tracking and Final Output Image

\subsubsection{Object Recognition}

After edge detection process mentioned in the previous section, extraction the key objects is executed. In this work, three types of object edges are recognized: straight, circular and annular edges. The Rake function is used in searching and identification of straight edges in the images using a number of parallel search lines covering the ROI as shown in Fig. 5.a. The Spoke function is used for identification of circular or annular features uses a number of lines, drawn from the center of the region to the outer boundary and ROI. The number of screech lines is determined specified by the angle between each line as shown in Fig. 5.b.
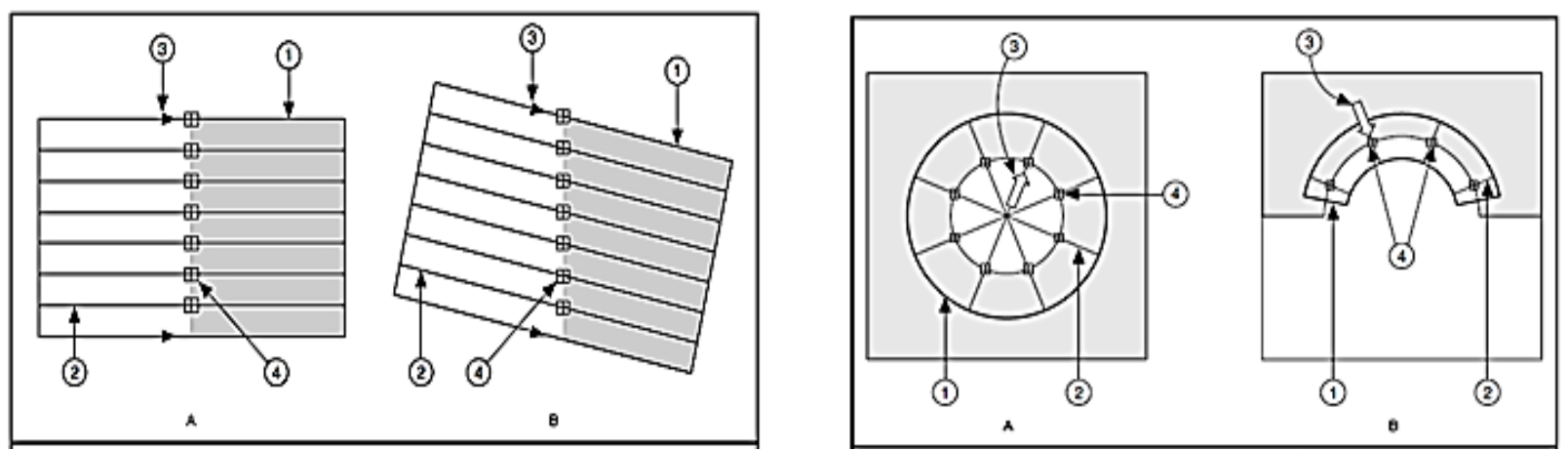

For All Figures: 1. Search Area; 2. Search Line; 3. Search Direction; 4 Edge Points Line.

Fig. 5 Detection and Recognition of Straight \& Circular Edges 


\subsection{Inspection}

Inspection module consists of two main stages: inspection and intermediate logic decisions. Inspection module consists of five main functions: measurement, counting, checking presence of parts or features, verification of proper part direction and proper surface coating as described before in Fig.1.

Measurements and checking part direction (guidance) in CAI-1 are based on edge detection and object recognition method described in section (3.4), to measure line distances, angles, areas the areas of geometric shapes. The generated results are in real world units for convenience inspection process.

Color segmentation is used for performing other inspection functions that involve: counting, checking features and parts presence, defects identification and surface Coating verification. The method is based on color segmentation to compare the color feature of each pixel with the color features of surrounding pixels or by training a color classifier to segment the image into color regions and separate color objects of interest from background clutter.

Colour segmentation is used to train the classifier by classifying sample images into new or existing classes. Based on those samples, the particle classifier can classify unknown samples into a known class. The main steps in developing classifier are :opening the example images; creating particle classes; testing the particle classifier; editing and saving the particle classifier.

The development of color classifier consists of two steps: training phase classification phase. In the training step, samples of the known region in the image containing the color that the classifier are learnt and labeled. For every sample added during the training phase, the color classifier calculates a color feature and assigns the associated class label to the feature. Eventually, all the trained samples (color feature with the label) added to the classifier are saved into a file which represents a trained color classifier.

After training the classifier, the regions in the image are classified into their corresponding classes for color identification. The color features of the sample under inspection are calculated to identify and classifies them among trained sample using a classification algorithms using the Minimum Mean Distance algorithm. As illustrated in Fig. 6, classification is performed into two main steps:

1.Segment an image into different color regions. Color segmentation consists of the following steps.

a. Moving an inspection window across the image to calculate the color feature of each pixel.

b. Compare the color feature for each inspection window with the color feature of neighboring windows.

c. Apply the color label from the pivot pixel in the neighboring window to the pivot pixel in the inspection window if the closest distance between the inspection window and a neighboring window is less than maximum distance, 


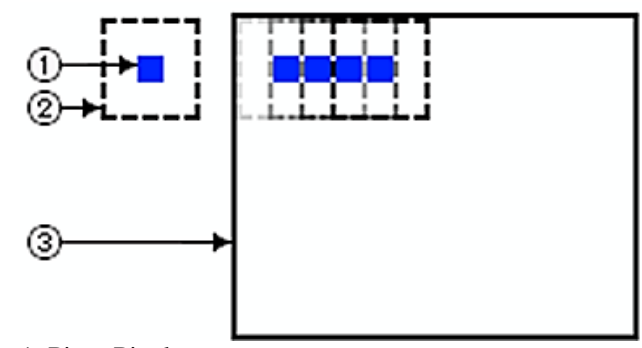

1. Pivot Pixel;

2. Inspection Window;

3. Image

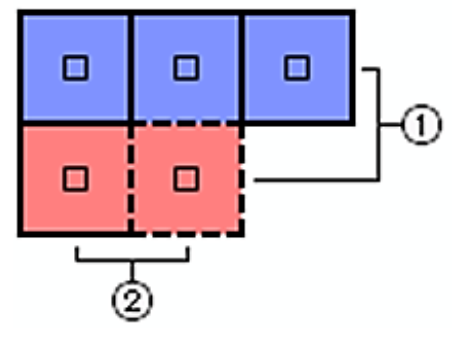

1. Distance Between Neighboring Color Feature Exceeds Maximum Distance

2. Distance Between Neighboring Color Feature Does Not Exceeds Maximum Distance

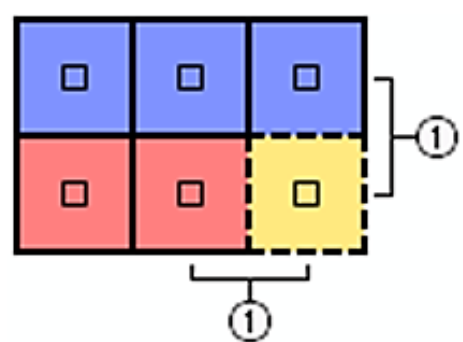

1. Distance Between Neighboring Color Feature Exceeds Maximum Distance

Fig.6 Colour Segmentation Technique

d. If the closest distance between the inspection window and a neighboring window is greater than maximum distance, use the color classifier to label the pivot pixel in the inspection window.

e. If the identification score for the inspection window is less than the minimum identification score, the color classification algorithm does not label the pivot pixel.

2.Filter the segmented image to eliminate regions that do not meet the specified size requirements. The Maximum distance refers to the maximum distance allowed between the color features of pivot pixels with the same color label. Maximum distance is calculated from the trained color classifier using the Equation 4.

Maximum Distance $=$ Distance Between Two Closest Trained Classes $\times \frac{\text { Step Size }}{\text { Window Size }}$

The inspection module proceeds to the intermediate measurement/ logic decisions step (4) this step, the generated results from previous inspection steps are used as inputs to perform the intermediate measurements inspection logic decisions. CAI-1 generates the measurement results obtained for each selected feature, the decision related to each part (Pass, Rework or Reject) and the final acceptance or rejection of the whole part assembly in all cases, failure of any part in the assembly to pass the inspection leads to generation of "Reject" and "Removal" from the inspection line. The intermediate measurements and generated decisions are performed according to developed logic and rules shown in the Appendix A (Table1) and implemented in the case study presented in section 4.

\subsection{Verification of Model Capabilities}

The developed model was tested to evaluate its accuracy by measuring three main entities attributes: lines, circles and angles. The system setup; image acquisition, calibration and processing were performed as mentioned in section (3.2). The selected ranges and sizes for each entity are shown in Table .1 .

The measured values were compared with the actual value. The errors were calculated in order to evaluate the model accuracy. According to the measurement results shown in Appendices B\&C, CAI-1 showed high measurement accuracy where it was $97 \%$ for line, 95\% for circle and $97.8 \%$. The charts in Appendix B show high decrease in the error ratio starting from $6 \mathrm{~mm}$ in line measurement and $8 \mathrm{~mm}$ for diameter. Accordingly, the recommended range for using CAI-1 in measurement applications and the corresponding accuracies are shown in Table (1). 
Table 1 CAI-1 Measurement Capabilities

\begin{tabular}{|l|c|c|c|c|c|}
\hline \multicolumn{1}{|c|}{ Feature } & $\begin{array}{c}\text { Selected } \\
\text { Range }\end{array}$ & Error Range & $\begin{array}{c}\text { Max. } \\
\text { Error }\end{array}$ & Max. Error \% & Accuracy \\
\hline Line Length (mm) & $6: 100$ & $-0.06:+0.16$ & 0.22 & $2.0 \%$ & $98.0 \%$ \\
\hline $\begin{array}{l}\text { Circle / Arc Diam. } \\
\text { (mm) }\end{array}$ & $5: 54$ & $+0.02:+0.28$ & 0.26 & $3.5 \%$ & $96.5 \%$ \\
\hline Angle (Deg.) & $5: 90$ & $-0.03:+0.19$ & 0.22 & $1.2 \%$ & $97.8 \%$ \\
\hline
\end{tabular}

\section{Case Study}

CAI-1 is a general inspection model that can be adopted for inspection of different assembly parts using their images as an input. The idea is based on feeding the model first with a reference image that contain proper features to use it as a reference in training the model for performing required inspection tasks. This case study illustrates the implementation of CAI-1 general automated inspection model. The model extracts lines, circles, angles and areas features and uses them in performing different inspection tasks. The model was simulated using a test sample consists of 11 pneumatic assembly parts shown in Fig.7 and Appendix C. The first part (P0) is used as a reference part that has complete assembly, acceptable measurements and surface coating. Other parts (P1:P10) have deferent defects and out-oftolerance dimensions, known in advance, but need to be identified by the model. Fig. shows the images of the used parts with their existing defects that will be identified by CAI-1.

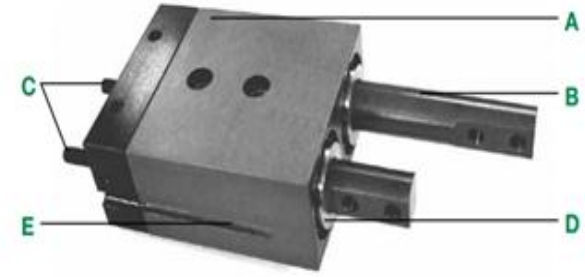

A. Body, B. Rods, C. Stop, D. Bushing, E. Sensor

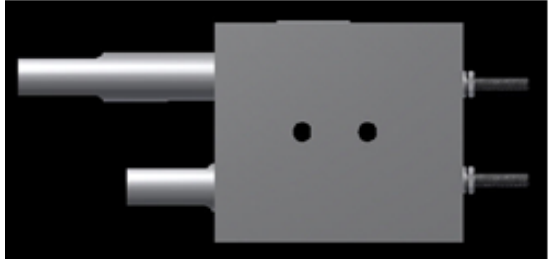

Inspected side

Fig. 7 Pneumatic Part

In this example, the following inspection tasks need to be performed tasks:

- Measurement of block length, height, holes sized and corner angle, diameters and length of both upper and lower rods and studs.

- Counting of existing holes

- Verification of presence of both holes and stud nuts.

- Verification of proper surface coating of the block part

- Checking of proper assembly direction.

The Model starts with image acquisition and calibration of the part images for making measurements in real world units $(\mathrm{mm})$ instead of pixel units. The model proceeds to locate and setup the part reference coordinates in the image as mentioned in section 3.3 and shown in Fig.8

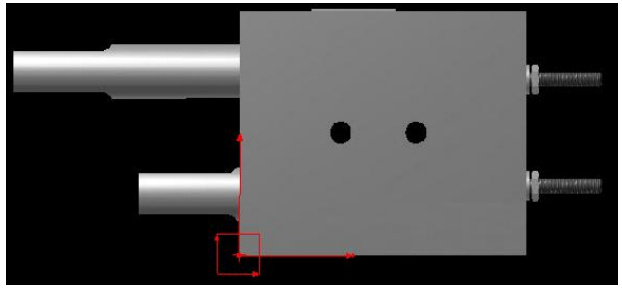

Fig.8 Locate and setup of part coordinates 
The model proceeds to detect existing edges via searching and identifying lines and circles features in the image as described in section (3.4) for performing Measurements, counting and checking part direction. The model applies image segmentation and filtering techniques to identify region and area characteristics for checking part presence and proper surface coating.

After simulating the model using the part images shown in Appendix $\mathrm{C}$ as inputs, the following features are detected: block, studs, rods and holes edges; and the surface region that reflect the surface coating. The obtained results were tabulated as shown in Appendix D, Where the first column is the inspection criteria, the second column include the acceptable tolerance values for each inspection part while the other column include all the generated inspection results for the 11 parts (P0:P10). The last row includes the generated final inspection result for each part. The inspected part assembly consists of three main parts: the block, the adjustment studs and the upper and lower rods.

The block inspection consists of measurement of five variables: block length, height, corner angle and inspection of surface coating. CAI-1 checks the presence and counts the existing holes and measure holes diameter and calculates the Ovality value in each diameter via measuring the diameter in two perpendicular directions to ensure that it is also within the limits. The logic rules shown in Appendix A are used for making required comparisons and calculations based on the extracted features. The block part will not be accepted unless all the previous conditions are met otherwise, the block will be rejected and the part will be removed for rework or rejection according to its condition. The rework decision is provided automatically by the CAI- 1 if the block length or height measured dimensions are above the allowable value provided that two conditions are existed corner angle is within the limit, one or more of the holes were not existed or its measurement is less than the allowable diameter The upper and lower adjustments studs inspection consist of inspection of thread diameters, length and nuts presence are checked. The inspected adjustment studs will pass only if both rods dimensions are met and both nuts exist in place. The upper and lower adjustments studs inspection consist of inspection of thread diameters, length and nuts presence are checked. The inspected adjustment studs will pass only if both studs diameters and length are within the tolerances and both nuts exist in place. Similarly, the upper and lower rods are inspected and they pass the inspection if rod diameters and length are within the acceptable tolerances.

The assembly direction verification is made to ensure the part is located in the proper direction. The condition for proper direction met only when: both rods exist and the length of upper rod > length of lower rod and that diameter of upper rod < Diameter of lower rod. Finally, acceptance of the whole assembly occurs when all of the above conditions are met (all inspection passed successfully).

The Generated results are automatically highlighted either with red or green colours according to the inspection condition. If the results are out of tolerance (for measurement inspection); or not identical ( for presence, surface coating and counting inspections); then they will be highlighted in red, while they will be highlighted with green if they are within the acceptable tolerances. Assemblies that passed all 25 inspection steps successfully had the inspection status ("Pass") highlighted in green as shown in last row while others that had one or more failed inspection steps had inspection status "Fail" and highlighted in red.

The findings illustrate successful implementation of CAI-1 model with the designed accuracy where:

1. The model could successfully differentiate between correct parts and defected / incomplete parts and generated the proper acceptance and rejection decisions faulty parts : $\mathrm{P}_{0}$ that has no defects or missing parts has passed the inspection while all other parts failed to pass the inspection for different reasons. 
2. Measurement and Identification of out of tolerance parts as in Upper stud diameter and lower stud length in $\mathrm{P}_{1}$; the upper stud diameter in $\mathrm{P}_{2}$; the upper stud diameter in $\mathrm{P}_{3}$; the Block height in $\mathrm{P}_{4}$; the Upper stud diameter in $\mathrm{P}_{5}$; the Block height and corner angle, Upper stud diameter and lower stud length and lower rod length in $\mathrm{P}_{6}$; the Upper stud diameter, lower stud length, lower rod length and diameter in $\mathrm{P}_{7}$; the Upper stud and upper rod diameters in $\mathrm{P}_{8}$; the LT hole diameter and Ovality, upper and lower rods diameters in $\mathrm{P}_{9}$; and the $\mathrm{RH}$ hole diameter and area, Upper stud diameter in $\mathrm{P}_{10}$.

3. Counting of existing parts / features as counting of existing holes in $\mathrm{P}_{1}, \mathrm{P}_{2}, \mathrm{P}_{3}$; and the upper stud nut in $\mathrm{P}_{4}$.

4. Checking Features / part presence as in identifying of the missing LH hole in $\mathrm{P}_{1} \& \mathrm{P}_{2}$; the missing $\mathrm{RH}$ hole in $\mathrm{P}_{3}$; and the missing upper stud nut in $\mathrm{P}_{4}$.

5. Identification of improper part guidance (part direction) as in $\mathrm{P}_{7}$.

6. Inspection of surface coating and identification of defected surface coating as in $\mathrm{P}_{4}$.

The inspection accuracy of CAI-1 was also validated to ensure its generalization capabilities via comparing the inspection results generated by the model with those obtained from physical measurement of the test parts as shown in Fig (9). The findings show that error range was $(-0.06:+0.16)$ for line distance measurement, $(+0.02:+0.28)$ for diameter measurement and $(-0.03:+0.18)$ for angle measurements. These results are consistent CAI-1 measurement capability shown in Table (1). The inspection time using CAI-1 model was also evaluated. To compare the inspection time required by CAI-1 and physical inspection methods, the following steps were used: Each part was inspected three times by three different inspectors in random order and the average total time required for inspecting each all parts was calculated (T1). The inspection cycle using CAI-1 was repeated three times for the same parts and the average total time (T2) was calculated too. By comparing both inspection times, the result shows that $\mathrm{T} 2$ is less than $0.05 \mathrm{~T} 1$.

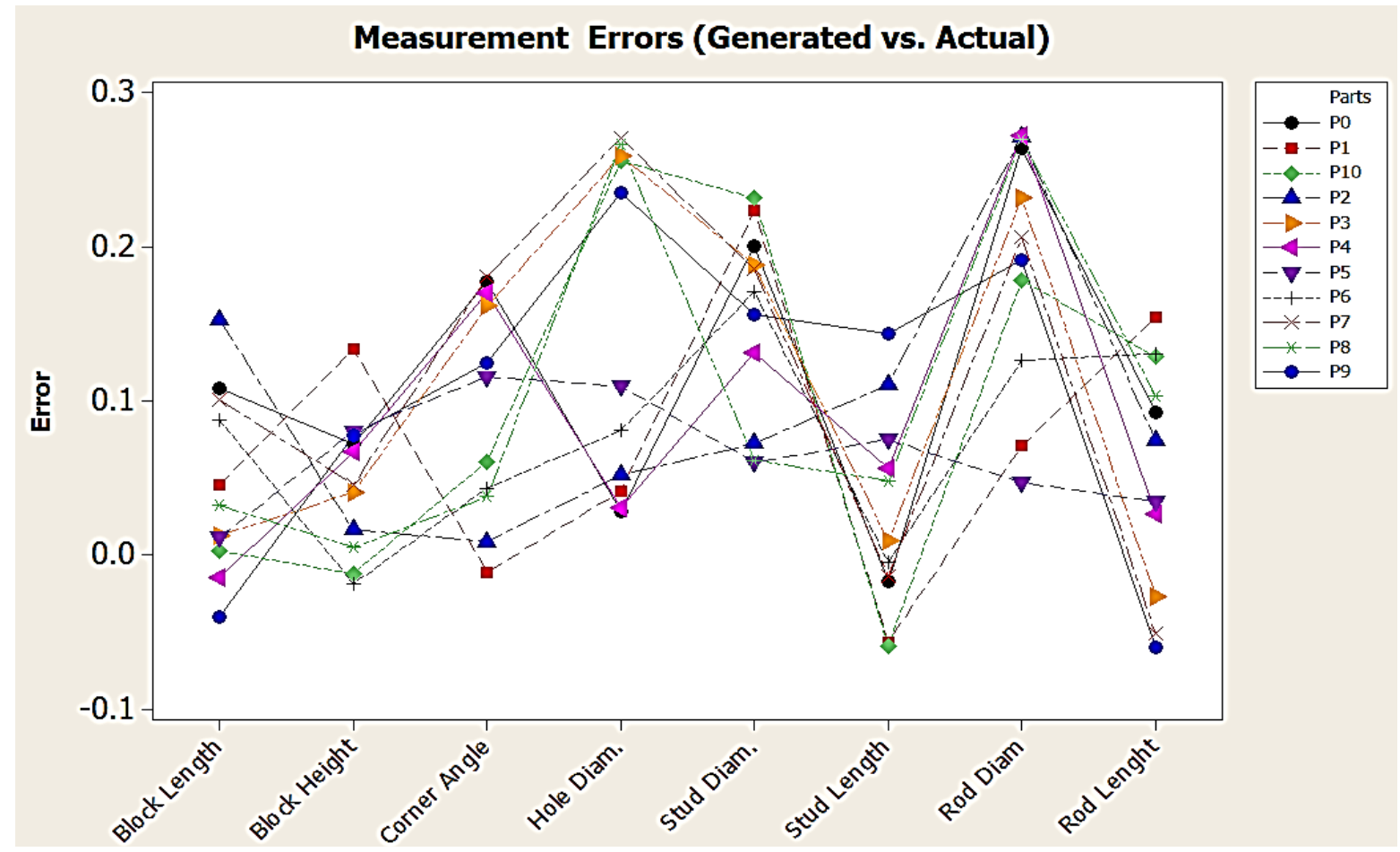

Fig. 9 Error Ranges (CAI-1 Generated vs. Actual Measurement) 


\section{Conclusion}

This paper presents new methodology for automated inspection of assembly parts using computer vision techniques based on feature extraction of lines, circles, angles and area characteristics in inspected part images. The developed model can be used as integrated system for measurement, counting, checking of part and feature presence and verification of proper part direction in the production lines. Digital images of parts under inspection are used as input and automatically generate the inspection results. Results include: measurement values, acceptance, rework or rejection for each part or feature and acceptance or Rejection decision of the whole assembly. The accuracy of the developed model was verified and validated. The measurement function accuracy were $98.0 \%, 96.5 \%$ and $97.8 \%$ for line, diameter and angle consequently. Other functions including counting, checking of part/feature presence, part direction and proper surface coating were performed without error.

The advantages of the developed CAI-1 model can be summarized into the following points: First, CAI-1 contributes in solving the automated visual inspection tasks besides performing logic decisions like pass, reject or rework the parts.: model is featured with the following advantages: support automated inspection models, ability to integrate in modern computer vision systems, high flexibility as it can be modified and adopted to use with other parts; high speed accuracy compared to traditional inspection systems. It can generate also inspection values and decisions for individual parts / features and the entire assembly as well.

CAI-1 can be integrated with an On Machines Inspection system and meets the needs to carry out inspection on the same machining center or in production line without the need for using inspection gauges or fixture changes. The benefits of this model include cost and time saving through decreasing lead-time required for gages and fixtures, minimizing the need for design, fabrication, maintenance of hard gages, fixtures \& equipment, elimination of non-value added operations such as lot inspection, sampling plans, receiving inspection, design, fabrication and maintenance of hard gages, and reworking nonconforming parts and reducing the inspection queue and time. CAI-1 support the changing from "reactive" inspection to "proactive" control by integrating quality control into product realization process, focusing resources on prevention of defects instead of detection in the end, utilizing real-time process knowledge and control [17].

\section{Future Work}

The efficiency of the proposed model can be enhanced via integration with artificial intelligence technologies like ANNs for enhancing the decisions generated from CAI-1 in terms of number and quality. Using the ANN would enhance the CAI-1 capability to deal with more complicated inputs and generate the desired decisions. Integrating CAI-1 with other advanced technologies such as probing strategy, error compensation, data analysis software and fixture design technology to create hybrid measurement systems.

\section{References}

[1] Turek, F. "Machine Vision Fundamentals: How to Make Robots", NASA Tech Briefs Magazine, 2011, Vol. 35. pp. 60-62.

[2] Abouelatta, O.B., "3D Surface Roughness Measurement Using a Light Sectioning Vision System", Proceedings of the World Congress on Engineering, 2010,Vol. I, WCE), pp.698-703. 
[3] Steger, C., Markus, U., and Christian W. (ed) Machine Vision Algorithms and Applications. Weinheim New York, 2008, p. 1.

[4] Zhao, F, Xu, X. \& Xie, S.Q. Computer-Aided Inspection Planning-The state of the art Computers in Industry, 2009 , Vol.60, pp. 453-466.

[5] Dutta, S. Pal, S.K., Mukhopadhyay, S Sen, R. "Application of digital image processing in tool condition monitoring: A Review" CIRP Journal of Manufacturing Science and Technology, 2013, 6, pp.212-232.

[6] Schmitt, R., Scholl, I., Cai1, Y., "Machine Vision System for Inline Inspection in Carbide Insert Production", Proceedings of the 36th International MATADOR Conference, 2010.

[7] Davies, E. R., "Machine Vision: Theory, Algorithms, Practicalities". Morgan Kaufmann., 2010, ISBN 0-12-206093-8.

[8] Gonzalez, R.C., Woods, R.E.(2.ed.), Digital Image Processing, Prentice- Hall Inc., New Jersey, 2002.

[9] CAI, S.; Liu,G. \& Zhang, B. "The research of the Appearance Detection System of Roller Bearings based on Image Technology", New Technology, 2009, Vol. 4, pp.42-43.

[10] Deng, S.;Cai, W. and Xu,Q. "Defect detection of bearing surfaces based on machine vision technique", International Conf. on Computer Application and System Modeling, 2010, pp. 548-554.

[11] Angrisania, L. Dapontea, P., Pietrosantoa, A. \& Liguorib, C. "An image-based measurement system for the characterization of automotive gaskets Measurement",1999, Vol. 25 , pp.169-181.

[12] Ayub, M. A. , Mohamed, A. B. and Esa, A. "In-line inspection of roundness using machine vision", 2nd International Conference on System Integrated Intelligence: Challenges for Product and Production Engineering Procedia Technology, 2014, Vol.15, pp.808 - 817 .

[13] Wu, B.; Zhang, F. and Xue, T. "Monocular-vision-based method for online measurement of pose parameters of weld stud Measurement", 2015, Vol.61, pp. 263-269

[14] Lu, R., Li,Y., and Yu, Q., "On-line measurement of the straightness of seamless steel pipes using machine vision technique", Sensors and Actuators, 2001, pp.95-101

[15] Ali, M. A., Kurokawa, S. and Uesugi, K. "Application of machine vision in improving safety and reliability for gear profile measurement", Machine Vision and Applications, 2014, Vol. 25, pp.1549-1559.

[16] Sills, K. • Bone, G. M. and Capson, D. Defect identification on specular machined surfaces, Machine Vision and Applications, 2014, Vol. 25, pp.377-388.

[17] Cho, M.; Lee, H.; Yoon, G. \& Choi, J.,“A computer-aided inspection planning system for on-machine measurement-Part II: Local inspection planning", KSME International Journal, 2004, Vol.18, pp.1358-1367. 


\section{Appendix A: Developed Inspection Logic \& Rules in CAI-1 \\ Table1 Logic Rules for features}

\begin{tabular}{|l|l|}
\hline Feature & Criteria \\
\hline \multirow{2}{*}{ Line } & $\begin{array}{l}\text { - Line exist } \\
\text { - Line is straight. } \\
\text { - Length within acceptable limits. }\end{array}$ \\
\hline Circle / Arc & $\begin{array}{l}\text { - Holes exist. } \\
\text { - Diameter size of the hole is within the acceptable limits. }\end{array}$ \\
\hline Angle & $\begin{array}{l}\text { - The angle between the selected edge is within acceptable limits } \\
\text { Region }\end{array}$ \\
\hline
\end{tabular}

\section{Table2 Implementation of CAI-1}

\begin{tabular}{|c|c|c|c|c|}
\hline $\begin{array}{c}\text { Detected } \\
\text { Feature }\end{array}$ & $\begin{array}{l}\text { Inspection } \\
\text { Function }\end{array}$ & $\begin{array}{c}\text { Part } \\
\text { under } \\
\text { Inspection }\end{array}$ & $\begin{array}{c}\text { Inspection } \\
\text { Task }\end{array}$ & Inspection Logic Rules \& Decision \\
\hline Line & Measurement & \multirow{6}{*}{$\begin{array}{l}\text { Block } \\
\text { Body }\end{array}$} & $\begin{array}{l}\text { Measurement } \\
\text { of Block } \\
\text { Length and } \\
\text { Height }\end{array}$ & \multirow{6}{*}{$\begin{array}{l}\text { 1. Accept Block if } \\
\text { - Block Length, Height and corner angle are within } \\
\text { acceptable limits. } \\
\text { - Both Holes exist. } \\
\text { - Number of holes = } 2 \\
\text { - Both Holes diameters \& ovalities are within acceptable } \\
\text { limits. } \\
\text { - Block surface coating is accepted. } \\
\text { 2. Rework Block if } \\
\text { - Block Length or Height > acceptable limits } \\
\text { - Corner angle is > or < acceptable limit by 0.5.Degree. } \\
\text { - Number of holes = } 1 \text { (missing hole need to be drilled) } \\
\text { - Any or Both Holes diameters < acceptable limit. } \\
\text { - Block surface coating is failed. } \\
\text { 3. Reject Block if: } \\
\text { - Block Length or Height < acceptable limit } \\
\text { - Any of Holes diameters or ovalities are > acceptable } \\
\text { limits }\end{array}$} \\
\hline Line & Measurement & & $\begin{array}{l}\text { Measurement } \\
\text { of Corner angle }\end{array}$ & \\
\hline Circle & $\begin{array}{l}\text { Check part } \\
\text { Presence }\end{array}$ & & $\begin{array}{l}\text { Identification } \\
\text { of Holes } \\
\text { presence. }\end{array}$ & \\
\hline Circle & Counting & & $\begin{array}{l}\text { Counting of } \\
\text { holes }\end{array}$ & \\
\hline Circle & Measurement & & $\begin{array}{l}\text { Measurement } \\
\text { of Holes } \\
\text { Ovality } \\
\end{array}$ & \\
\hline Region & $\begin{array}{l}\text { Inspection of } \\
\text { surface } \\
\text { Coating }\end{array}$ & & $\begin{array}{l}\text { Inspection of } \\
\text { block surface } \\
\text { coating c }\end{array}$ & \\
\hline Line & Measurement & \multirow{2}{*}{$\begin{array}{l}\text { Upper \& } \\
\text { Lower } \\
\text { Adjustmen } \\
\text { t Studs }\end{array}$} & $\begin{array}{l}\text { Stud diameters } \\
\text { \& lengths }\end{array}$ & \multirow{2}{*}{$\begin{array}{l}\text { 4. Accept Adjustment if } \\
\text { - Stud diameters \& lengths are within acceptable limits } \\
\text { - Both nuts exist } \\
\text { 5. Reject adjustment studs if : } \\
\text { - Stud diameters or lengths are out of limits; or } \\
\text { - Any or both nuts are missing }\end{array}$} \\
\hline Region & $\begin{array}{l}\text { Check part } \\
\text { presence }\end{array}$ & & Nuts exist & \\
\hline $\begin{array}{l}\text { Line } \\
\text { Edge }\end{array}$ & Measurement & $\begin{array}{l}\text { Upper \& } \\
\text { Lower } \\
\text { Rod }\end{array}$ & $\begin{array}{l}\text { ROD diameters } \\
\& \text { lengths }\end{array}$ & $\begin{array}{l}\text { 6. Accept Rod if } \\
\text { - Stud diameters \& lengths are within acceptable limits } \\
\text { 7. Reject Rod if : } \\
\text { - Stud diameters or lengths are out of limits }\end{array}$ \\
\hline $\begin{array}{l}\text { Line } \\
\text { Edge }\end{array}$ & $\begin{array}{l}\text { Check } \\
\text { assembly } \\
\text { direction }\end{array}$ & $\begin{array}{c}\text { Whole } \\
\text { Assembly }\end{array}$ & $\begin{array}{l}\text { Check of } \\
\text { proper } \\
\text { Assembly } \\
\text { Direction }\end{array}$ & $\begin{array}{l}\text { 8. Accept Part Direction if } \\
\text { - Upper Rod length > Lower Rod length. } \\
\text { 9. Remove Part and Reposition if } \\
\text { - Upper Rod length < Lower Rod length. (The part is } \\
\text { rotated). }\end{array}$ \\
\hline
\end{tabular}




\section{Appendix B: CAI-1 Inspection Test Results}
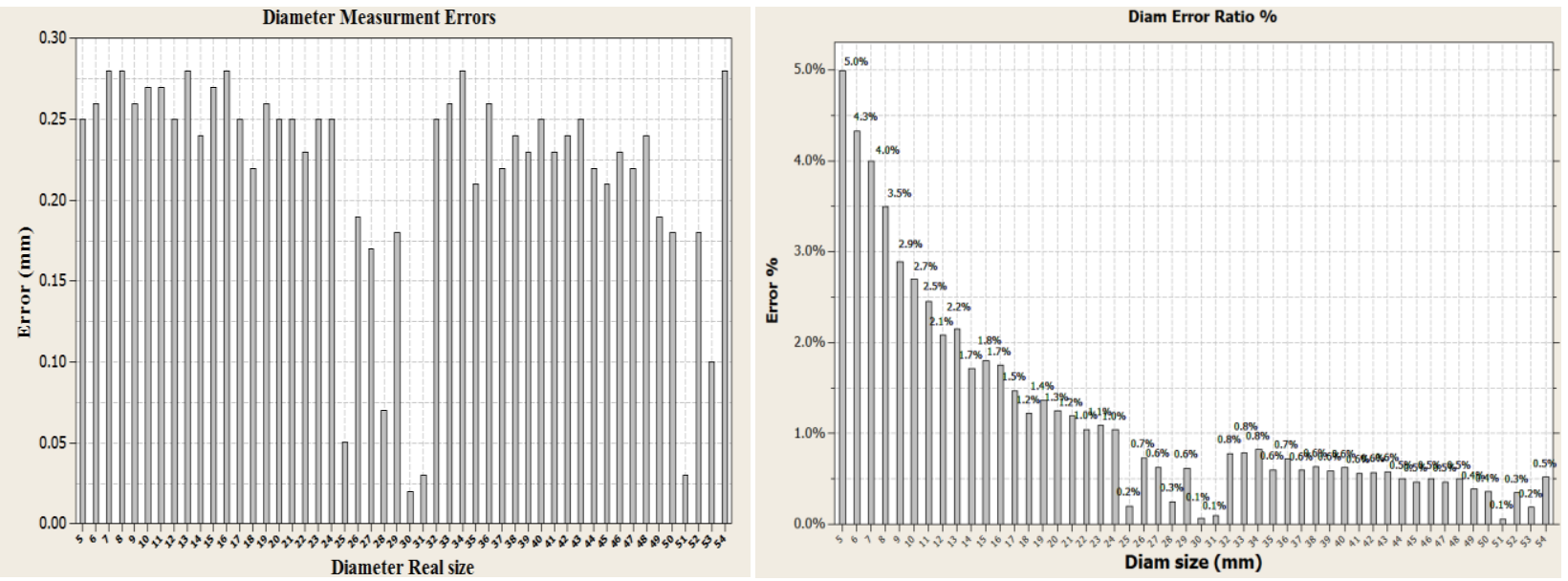

Fig. b.1 Diameter Measurement Results Errors
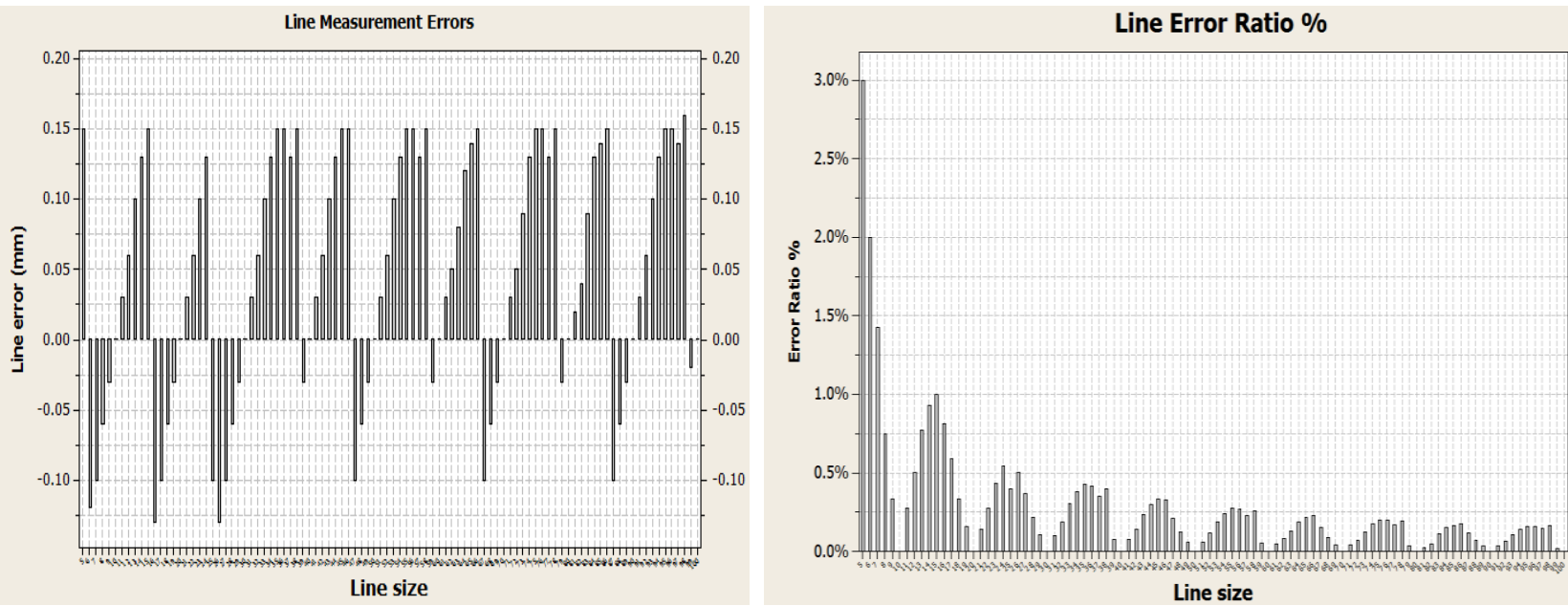

Fig. b.2 Line Measurement Results Errors
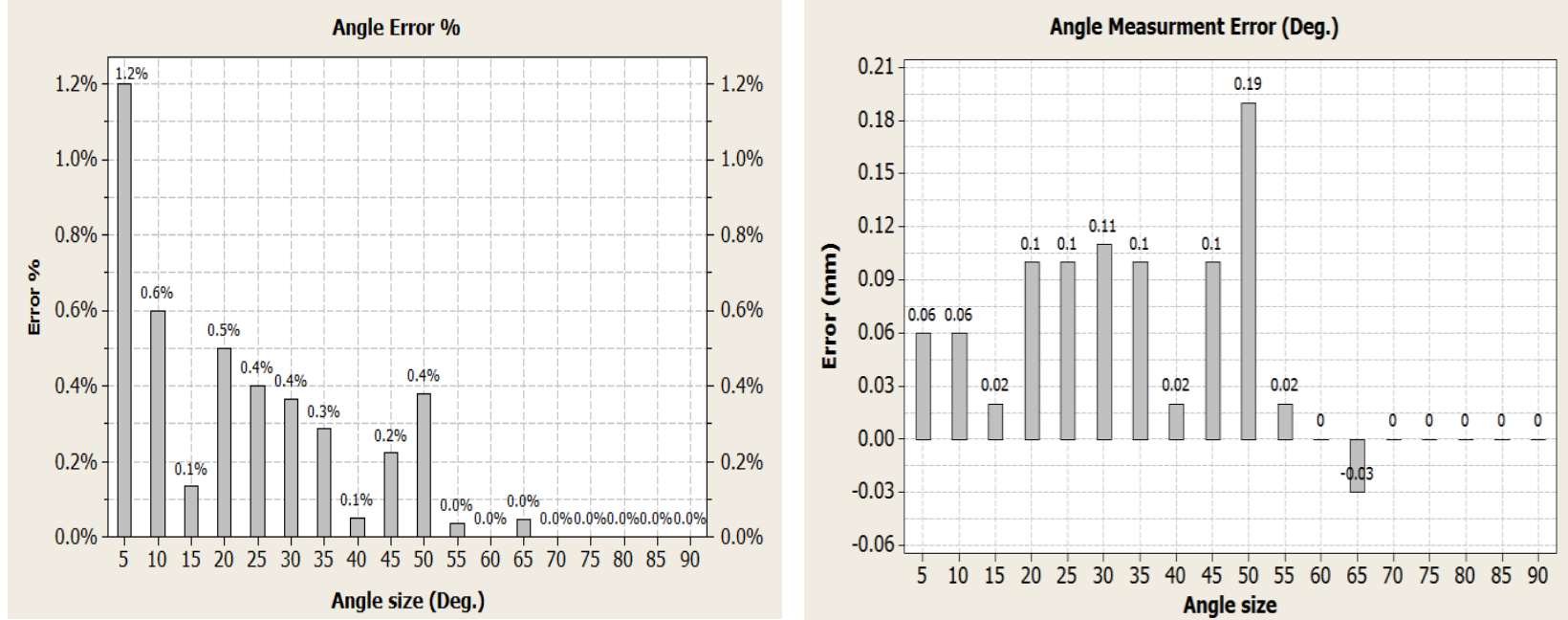

Fig. b.3 Angle Measurement Results Errors 


\section{Appendix C: Images of Inspected Parts}

\begin{tabular}{|c|c|c|}
\hline Part name & Part Image & Existing Defects \\
\hline Po & $\bullet$ & - Perfect part - No defect \\
\hline P1 & • & $\begin{array}{l}\text { - One missing hole, Upper stud diameter \& Lower } \\
\text { stud length < acceptable limits }\end{array}$ \\
\hline P2 & $\bullet$ & $\begin{array}{l}\text { - One missing hole, } \\
\text { - } \quad \text { Upper stud diameter }<\text { Acceptable limits }\end{array}$ \\
\hline P3 & • & $\begin{array}{l}\text { - One missing hole, } \\
\text { - Upper stud diameter }<\text { Acceptable limits }\end{array}$ \\
\hline P4 & • & $\begin{array}{l}\text { - } \quad \text { Block length }<\text { acceptable limits } \\
\text { - Upper nut is missing } \\
\text { - } \quad \text { Surface coating defect. }\end{array}$ \\
\hline P5 & $\bullet$ & - Upper stud diameter $<$ Acceptable limits \\
\hline P6 & $\bullet$ & $\begin{array}{l}\text { - Block Height \& Corner angle \& Upper stud } \\
\text { diameter \& Lower stud length }<\text { Acceptable } \\
\text { Height }\end{array}$ \\
\hline P7 & & $\begin{array}{l}\text { - Upper stud diameter \& Lower stud length and } \\
\text { Diameter < Acceptable Height. } \\
\text { - The part direction is wrong. }\end{array}$ \\
\hline P8 & $\bullet$ & $\begin{array}{l}\text { - Upper stud diameter \& Upper rod Diameter < } \\
\text { Acceptable Height. }\end{array}$ \\
\hline P9 & $\bullet$ & $\begin{array}{l}\text { - Hole diameter and Ovality \& Upper rod Diameter } \\
>\text { Acceptable limits, }\end{array}$ \\
\hline P10 & $\bullet$ & $\begin{array}{l}\text { - Hole } 2 \text { Diameter, area \& Ovality > Acceptable } \\
\text { limits } \\
\text { - Upper stud Diameter > Acceptable limits }\end{array}$ \\
\hline
\end{tabular}


Appendix D: Inspection Results

\begin{tabular}{|c|c|c|c|c|c|c|c|c|c|c|c|c|}
\hline \multirow{2}{*}{$\begin{array}{l}\text { Inspection } \\
\text { Criteria }\end{array}$} & \multirow{2}{*}{$\begin{array}{l}\text { Nomina } \\
\text { I Value }\end{array}$} & \multicolumn{11}{|c|}{ Part name } \\
\hline & & $\mathbf{P}_{0}$ & $\mathbf{P}_{1}$ & $\mathbf{P}_{2}$ & $\mathbf{P}_{3}$ & $\mathbf{P}_{4}$ & $\mathbf{P}_{5}$ & $\mathbf{P}_{6}$ & $\mathbf{P}_{7}$ & $\mathbf{P}_{8}$ & $\mathbf{P}_{9}$ & $\mathbf{P}_{10}$ \\
\hline 1. Block Length & $\begin{array}{l}247.5: \\
248.5\end{array}$ & 248.36 & 248.37 & 248.37 & 248.37 & 247.3 & 248.37 & 248.36 & 248.37 & 248.37 & 248.36 & 248.37 \\
\hline $\begin{array}{l}\text { 2. Block } \\
\text { Height }\end{array}$ & 195:196 & 195.66 & 195.66 & 195.66 & 195.66 & 195.66 & 195.66 & 190.76 & 195.66 & 195.66 & 195.65 & 195.66 \\
\hline $\begin{array}{l}\text { 3. Block } \\
\text { Corner angle }\end{array}$ & $\begin{array}{c}89.5: \\
90.0\end{array}$ & 89.5 & 89.5 & 89.5 & 89.5 & 89.5 & 89.5 & 89.4 & 89.5 & 89.5 & 89.5 & 89.5 \\
\hline $\begin{array}{l}\text { 4. Hole } \\
\text { Presence }\end{array}$ & $\begin{array}{c}\text { Pass / } \\
\text { Fail } \\
\end{array}$ & Pass & Fail & Fail & Fail & Pass & Pass & Pass & Pass & Pass & Pass & Fail \\
\hline $\begin{array}{l}\text { 5. No. of } \\
\text { Holes }\end{array}$ & 2 & 2 & 1 & 1 & 1 & 2 & 2 & 2 & 2 & 2 & 2 & 2 \\
\hline 6.Hole 1 Area & $255: 258$ & 257.59 & Fail & Fail & 257.59 & 257.59 & 257.59 & 257.59 & 257.59 & 257.59 & 255.62 & 257.59 \\
\hline 7.Hole 2 Area & $255: 258$ & 255.62 & 255.6 & 255.6 & Fail & 255.6 & 255.6 & 255.6 & 255.6 & 255.6 & 255.6 & 300.0 \\
\hline $\begin{array}{l}\text { 8. Hole } 1 \\
\text { Diam. }(x)\end{array}$ & $\begin{array}{l}17.8: \\
17.9\end{array}$ & 17.88 & Fail & Fail & 17.89 & 17.89 & 17.89 & 17.89 & 17.89 & 17.89 & 19.93 & 17.89 \\
\hline $\begin{array}{l}\text { 9. Hole } 1 \\
\text { Diam. }(y)\end{array}$ & $\begin{array}{l}17.8: \\
17.9 \\
\end{array}$ & 17.88 & Fail & Fail & 17.88 & 17.88 & 17.88 & 17.88 & 17.88 & 17.88 & 17.88 & 17.88 \\
\hline $\begin{array}{l}\text { 10. Hole } 1 \\
\text { Ovality }\end{array}$ & $\begin{array}{l}0.00: \\
0.10\end{array}$ & 0 & 0 & 0 & 0.01 & 0.01 & 0.01 & 0.01 & 0.01 & 0.01 & 2.05 & 0.01 \\
\hline $\begin{array}{l}\text { 11. Hole } 2 \\
\text { Diam. }(\mathrm{x})\end{array}$ & $\begin{array}{l}17.8: \\
17.9\end{array}$ & 17.89 & 17.89 & 17.89 & Fail & 17.89 & 17.89 & 17.89 & 17.89 & 17.89 & 17.89 & 20.92 \\
\hline $\begin{array}{l}\text { 12. Hole } 2 \\
\text { Diam.(y) }\end{array}$ & $\begin{array}{l}17.8: \\
17.9\end{array}$ & 17.88 & 17.86 & 17.86 & Fail & 17.86 & 17.86 & 17.86 & 17.86 & 17.86 & 17.86 & 20.17 \\
\hline $\begin{array}{l}\text { 13. Hole } 2 \\
\text { Ovality }\end{array}$ & $\begin{array}{l}0.00: \\
0.10\end{array}$ & 0.01 & 0.03 & 0.03 & 0.00 & 0.03 & 0.03 & 0.03 & 0.03 & 0.03 & 0.03 & 0.75 \\
\hline $\begin{array}{l}\text { 14. Upper } \\
\text { Stud Diam. }\end{array}$ & $\begin{array}{l}11.7: \\
11.9\end{array}$ & 11.85 & 11.64 & 11.64 & 11.64 & 11.75 & 11.64 & 11.64 & 11.64 & 11.64 & 11.77 & 11.64 \\
\hline $\begin{array}{l}\text { 15. Upper } \\
\text { Stud Length }\end{array}$ & $\begin{array}{l}64.0: \\
65.0\end{array}$ & 64.22 & 64.15 & 64.15 & 64.15 & 64.1 & 64.15 & 64.09 & 64.15 & 64.15 & 64.17 & 64.15 \\
\hline $\begin{array}{l}\text { 16. Upper } \\
\text { Stud Nut } \\
\text { Presence }\end{array}$ & $\begin{array}{l}\text { Pass / } \\
\text { Fail }\end{array}$ & Pass & Pass & Pass & Pass & Fail & Pass & Pass & Pass & Pass & Pass & Pass \\
\hline $\begin{array}{l}\text { 17. Lower } \\
\text { Stud Diam. }\end{array}$ & $\begin{array}{l}11.7: \\
11.9\end{array}$ & 11.83 & 11.83 & 11.82 & 11.86 & 11.86 & 11.88 & 11.85 & 11.82 & 11.83 & 11.84 & 11.81 \\
\hline $\begin{array}{l}\text { 18. Lower } \\
\text { Stud Length }\end{array}$ & $\begin{array}{l}64.0: \\
65.0\end{array}$ & 64.13 & 54.22 & 64.17 & 64.17 & 64.17 & 64.15 & 63.92 & 64.17 & 64.17 & 64.15 & 64.17 \\
\hline $\begin{array}{l}\text { 19. Lower } \\
\text { Stud Nut } \\
\text { Presence }\end{array}$ & $\begin{array}{l}\text { Pass / } \\
\text { Fail }\end{array}$ & Pass & Pass & Pass & Pass & Pass & Pass & Pass & Pass & Pass & Pass & Pass \\
\hline $\begin{array}{l}\text { 20. Upper Rod } \\
\text { Length }\end{array}$ & $195: 196$ & 195.71 & 195.72 & 195.72 & 195.72 & 195.72 & 195.72 & 195.69 & 195.72 & 195.72 & 195.72 & 195.72 \\
\hline $\begin{array}{l}\text { 21. Upper Rod } \\
\text { Diam. }\end{array}$ & $\begin{array}{l}32.3: \\
32.4\end{array}$ & 32.39 & 32.39 & 32.39 & 32.39 & 32.39 & 32.39 & 32.39 & 32.39 & 32.47 & 32.41 & 32.39 \\
\hline $\begin{array}{l}\text { 22. Lower } \\
\text { Rod Length }\end{array}$ & $86: 87$ & 86.88 & 86.85 & 86.85 & 86.85 & 86.85 & 86.85 & 87.08 & 83.66 & 86.87 & 86.85 & 86.85 \\
\hline $\begin{array}{l}\text { 23. Lower } \\
\text { Rod Diam. }\end{array}$ & $\begin{array}{c}32.4: \\
32.5\end{array}$ & 32.45 & 32.47 & 32.47 & 32.47 & 32.47 & 32.47 & 32.47 & 32.31 & 32.47 & 32.52 & 32.47 \\
\hline $\begin{array}{l}\text { 24. Assembly } \\
\text { Direction }\end{array}$ & $\begin{array}{c}\text { Pass / } \\
\text { Fail }\end{array}$ & Pass & Pass & Pass & Pass & Pass & Pass & Pass & Fail & Pass & Pass & Pass \\
\hline $\begin{array}{l}\text { 25. Surface } \\
\text { Treatment }\end{array}$ & $\begin{array}{l}\text { Pass / } \\
\text { Fail }\end{array}$ & Pass & Pass & Pass & Pass & Fail & Pass & Pass & Pass & Pass & Pass & Pass \\
\hline Inspection Statu & & Pass & Fail & Fail & Fail & Fail & Fail & Fail & Fail & Fail & Fail & Fail \\
\hline
\end{tabular}

